\title{
Research Activities of Nanodimensional Standards Using Atomic Force Microscopes, Transmission Electron Microscope, and Scanning Electron Microscope at the National Metrology Institute of Japan
}

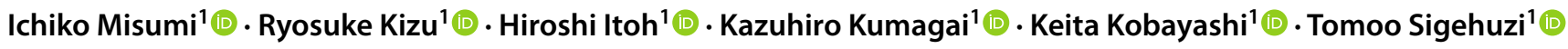

Received: 4 August 2021 / Revised: 26 September 2021 / Accepted: 5 October 2021 / Published online: 4 November 2021

(c) The Author(s) 2021, corrected publication 2022

\begin{abstract}
With the progress in nanotechnology, the importance of nanodimensional standards is increasing. Realizing nanodimensional standards requires multiple types of high-precision microscopy techniques. The National Metrology Institute of Japan (NMIJ), one of the research domains in the National Institute of Advanced Industrial Science and Technology (AIST), is developing nanodimensional standards using atomic force, transmission electron, and scanning electron microscopes. The current status of nanodimensional standards in NMIJ is introduced herein.
\end{abstract}

Keywords Nanodimensional standards · Calibration · Uncertainty · AFM · TEM - SEM

\section{Introduction}

With the progress in nanotechnology, the importance of dimensional nanometrology, which is the precise measurement of the dimensions of developed nanostructures, is increasing. To perform nanometrology precisely, highly reliable nanodimensional standards technology is required. Realizing nanodimensional standards technology requires developing multiple types of high-precision microscope technologies and an effective sample preparation technology for microscopes. National Metrology Institutes (NMIs) worldwide have developed various nanodimensional standards, such as the pitch of a one-dimensional grating [1, 2], pitch of a two-dimensional grating [3], step height [4, 5], linewidth [6, 7], degree of surface roughness [8], and size of standard nanoparticles [9, 10], as measured using several types of instruments [11].

The National Metrology Institute of Japan (NMIJ), the NMI in Japan and one of the seven research domains in the National Institute of Advanced Industrial Science and Technology (AIST), was reorganized in April 2020, and the

Ichiko Misumi

misumi.i@aist.go.jp

National Institute of Advanced Industrial Science and Technology, AIST Tsukuba Central 5, 1-1-1 Higashi, Ibaraki 305-8565 Tsukuba, Japan
Nanodimensional Standards Group was established. The group is working to develop nanodimensional standards using several types of microscopes, such as metrological atomic force microscopes (metrological AFMs) [12-14], scanning probe microscopes (SPMs) [15, 16], a scanning electron microscope (SEM) [17, 18], and a transmission electron microscope (TEM) [19]. Moreover, using these technologies, we aim to contribute to society through calibration services, the distribution of certified reference material (CRM) [20], standardization [21, 22], and collaboration (both inside and outside the group). Herein, the current status of the activities of the group is described.

\section{Atomic Force Microscope}

\subsection{Metrological Atomic Force Microscope}

This subsection introduces a new metrological AFM. We previously developed atomic force microscopes equipped with high-resolution laser interferometers (metrological AFMs) [22-24]. We also provided calibration services for measuring the pitches of one-dimensional [23] and twodimensional [23] gratings, step height [24], and surface roughness [25] using metrological AFMs. Recently, we developed a new metrological AFM and started a linewidth calibration service. Building upon our previous work, we developed a metrological AFM with a tilt probe mechanism 


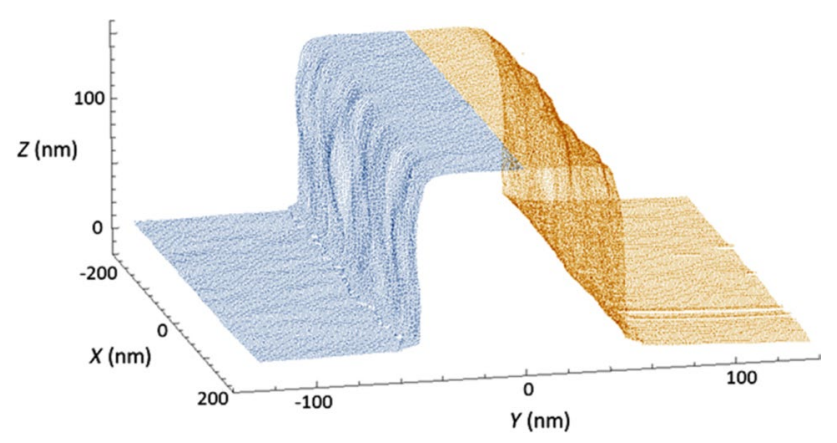

Fig. 1 Three-dimensional shape measurement of a silicon line pattern using a metrological atomic force microscope with a tilt probe mechanism (tilting-mAFM)

(tilting-mAFM) that extends the 3D nanomeasurement function for high-resolution topography measurement on the surface of the vertical side wall and traceable measurement of nanodevice linewidth [24]. In the new mAFM, the tip of the tilting-mAFM probe is tilted vertically up to $16^{\circ}$ so that it can be traced in contact with the vertical sidewalls of the nanoscale line patterns. Conventional AFM probes cannot reach the vertical sidewall due to the finite cone angle. The displacement of the probe was measured on three axes using a high-resolution laser interferometer that was traceable to the national standard of length. The parallel spring construction of the centrally symmetric 3D scanner enabled probe scanning with very low crosstalk between axes. A unique technique was also developed to scan the vertical sidewalls that showed high reproducibility in the scan profile and side wall angle measurements. Further, the 3D measurements of line patterns were demonstrated, and data from both sidewalls were combined with subnanometer accuracy (Fig. 1).

The linewidth, which is the critical dimension (CD) of a nanoscale line pattern, was calibrated using the tiltingmAFM [26]. The tilting probe allows the scanning of the vertical sidewalls of the line pattern. In the measurement of the linewidth, two measurements on each side of the line pattern were made using the tilting-mAFM; the two datasets were stitched; and the complete shape of the line pattern was reconstructed. Moreover, the $\mathrm{CD}$ based on this pattern was measured. To validate this $\mathrm{CD}$ calibration procedure, a linewidth standard with subnanometer-scale uncertainty [27] was used as the measurement sample. The CD calibrated with tilting-mAFM was $111.2 \mathrm{~nm}$, and the expanded uncertainty was $1.0 \mathrm{~nm}$. This uncertainty is the minimum uncertainty observed during CD measurements reported using a tilted AFM. Moreover, the difference between this $C D$ value and the $C D$ reference value was only $0.2 \mathrm{~nm}$. This result shows that a tilting-mAFM can be used for CD measurements with single nanometer accuracy. We are currently providing the calibration service of $C D$ using the tilting-mAFM.

\subsection{AFM Probe Characterizer}

An AFM can measure the nanostructures of size from several hundred nanometers to atomic structures as threedimensional images, which cannot be seen with an optical microscope. However, artifacts (shape errors) arise in AFM measurements because AFM probes are several nanometers to tens of nanometers in size. To realize highly accurate nanomeasurement without shape errors, we developed a probe characterizer to be used with an AFM [25]. Figure 2 shows a schematic of the AFM probe characterizer [15]. The AFM probe characterizer uses thin plate knife edges to measure the cross-sectional shape of the AFM probe and multiple gaps of different sizes to measure changes in the aspect ratio of the probe. The aspect ratio of the probe can be measured from the characterizer's AFM image, and the relationship between the width and length of the probe can be displayed (Fig. 3) [15]. The probe shape function allows the shape correction of the nanomaterial morphology. Based on this technology, "ISO 13095: 2014 Surface Chemical Analysis-Atomic force microscopy - Procedure for in situ characterization of AFM probe shank profile used for nanostructure measurement" [21] has been established by ISO TC 201 SC9 (ISO 201 Committee, surface chemistry analysis, scanning probe microscope).

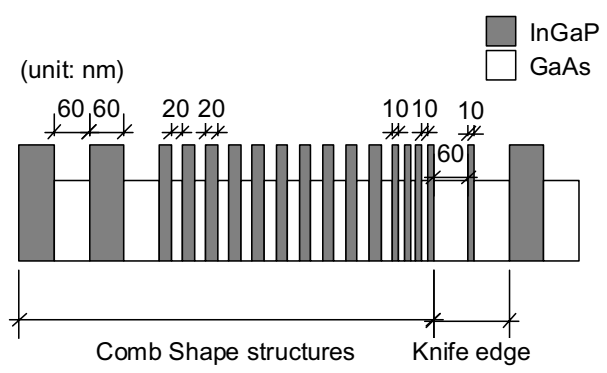

Fig. 2 Schematic of the probe characterizer

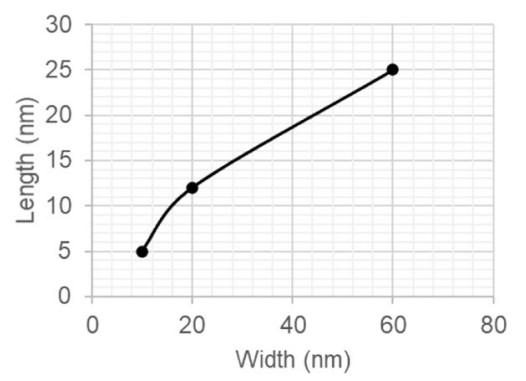

Fig. 3 An example of the probe shape analyzed from the averaged line profile of the probe characterizer 


\section{Scanning ew32 Microscope}

As with other microscope technologies, spatial image resolution is one of the greatest concerns for both equipment manufacturers and the users of SEMs. Considerable care must be taken when the image resolution is evaluated because resolution is one of the important indicators of SEM equipment performance. The spatial resolution of an SEM depends on the diameter of the primary electron (PE) beam, image contrast, and signal-to-noise ratio. There are several evaluation methods for spatial resolution, e.g., the PE beam diameter evaluation [28], gap method [29], Fourier transform (FT) method [30], derivative (DR) method [31], contrast-gradient (CG) method [32], and correlation method [33]. Each of these methods has its advantages and disadvantages. For example, the gap method, which is a simple and common method of specifying the resolution of an SEM image that measures the smallest visible gap between two particles in an image, remains unclear and the reproducibility is low because it detects the smallest gap with the human eye. ISO TC 202, Microbeam Analysis, Subcommittee SC 4, Scanning electron microscopy has standardized the methods of image resolution measurement by building a consensus among related industries and research areas. Further, a published technical specification provides image analysis procedures for FT, DR, and CG methods [34]. Recently, the committee has begun to develop a new international standard for quantitatively determining image sharpness by adopting the DR method from the three methods of technical specifications [35].

The main purposes of evaluating image resolution are to evaluate the device performance and to visualize the SEM operator's skill. The image sharpness value is the value given to each SEM image that reflects all the factors related to image formation. According to the SEM image formation principle, an SEM image depends on the sample, electron optics system, and observation conditions of the device. Therefore, when performing image sharpness evaluation for the above purposes, using the same sample and fixing at least the parameter of the sample are necessary. Considering the requirements and convenience of technical specifications, the sample for image sharpness evaluation should meet the following characteristics: (1) Magnification calibration and image sharpness evaluation can be performed on the same sample; (2) No image contrast abnormality due to the edge effect or charging should occur; (3) The image contrast is stable over a wide accelerating voltage range; and (4) The sample has a particle structure. Considering the above four conditions, a sample for image sharpness evaluation was designed with a dot array in which tungsten dots are arranged in a grid pattern at a constant pitch $d$ on a silicon substrate as the basic structure and is manufactured via a semiconductor process (Fig. 4) [17, 18]. Tungsten dots in the SEM image show a bright contrast against the silicon substrate, and the contrast is stable in the accelerating voltage range of general-purpose SEMs. The dot pitch is certified, and it can be used to determine the pixel size of an SEM image, in other words, to calibrate the magnification. The image sharpness value can be obtained in the dimension of length by performing image sharpness evaluation using the DR method on the image whose magnification has been calibrated using the dot array. Three types of dot arrays, i.e., $\mathrm{A}, \mathrm{B}$, and $\mathrm{C}$ with different dot diameters and pitches, are arranged, and these can be used in a wide range of magnifications of conventional SEMs. This sample has undergone a certification process at NMIJ, AIST, and is supplied as a CRM "NMIJ CRM 5207-a Tungsten Dot array" with dot pitches as the certified values [20]. The SEM images of NMIJ CRM 5207-a are shown in Fig. 5.

The uncertainty of the dot pitch of the CRM was evaluated [17]. The major uncertainty components are the homogeneity of samples, fluctuation of the dot pitch within ten samples, fluctuation of the dot pitch within three fields of view, fluctuation of the dot pitch within 11 rows in a secondary electron image, fluctuation of the dot pitch within ten intervals in a row, fluctuation of the dot pitch within repeated image acquisitions, microscale for magnification calibration, and image digitization. Table 1 shows the uncertainty budget of the dot-pitch values for Array $\mathrm{B}$, and Table 2 shows the certified values and expanded uncertainties.

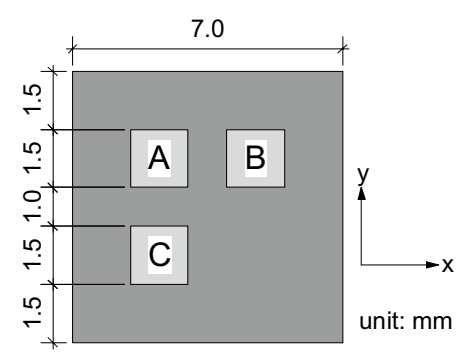

(a)
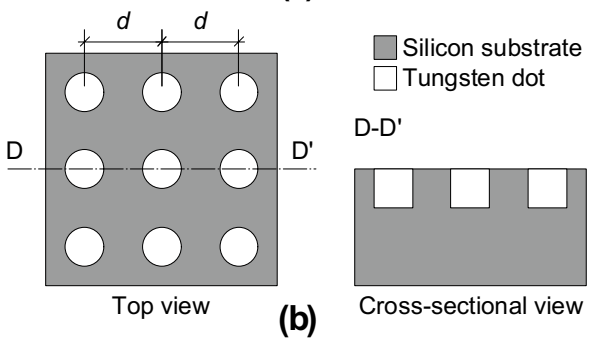

(b)

Fig. 4 a Whole view of the NMIJ CRM 5207-a; b schematics of the tungsten dot-array part 


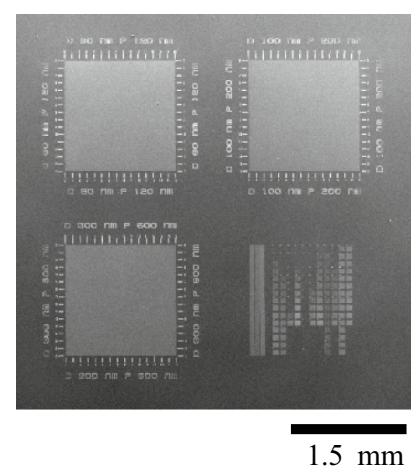

(a)

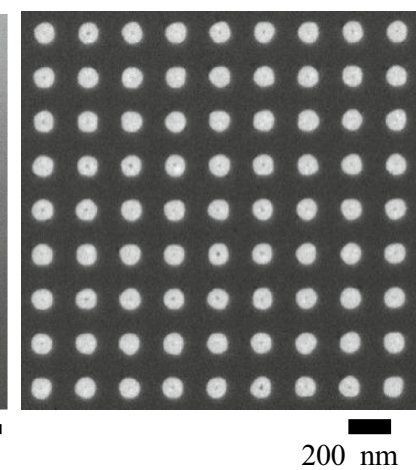

(b)
Fig. 5 SEM images of NMIJ CRM 5207-a: a whole view; b the tungsten dot-array part (Array B)

Table 1 Uncertainty budget of the dot-pitch values for Array B

\begin{tabular}{lll}
\hline Name of uncertainty component & \multicolumn{2}{l}{$\begin{array}{l}\text { Uncertainty component } \\
(\mathrm{nm})\end{array}$} \\
\cline { 2 - 3 } & $x$-direction & $y$-direction \\
\hline Homogeneity & 0.026 & 0.060 \\
Sample & 0.006 & 0.054 \\
Field of views (FOV) & 0.007 & 0.003 \\
Rows & 0.005 & 0.006 \\
Intervals in a row & 0.024 & 0.024 \\
Repeated image acquisitions & 0.002 & 0.001 \\
The microscale for magnification calibra- & 0.995 & 0.995 \\
$\quad$ tion & & \\
Image digitization & 0.672 & 0.672 \\
Combined standard uncertainty & 1.202 & 1.204 \\
Expanded uncertainty (coverage factor, & 2.4 & 2.4 \\
$\quad k=2)$ & & \\
\hline
\end{tabular}

Table 2 Certified values and expanded uncertainties of the dot pitches

\begin{tabular}{llll}
\hline Dot array & \multicolumn{2}{l}{$\begin{array}{l}\text { Dot pitch (nm) (certi- } \\
\text { fied value) }\end{array}$} & $\begin{array}{l}\text { Expanded uncertainty } \\
\text { (coverage factor, } k=2) \\
(\mathrm{nm})\end{array}$ \\
\hline A & $x$ & 119.0 & 1.5 \\
& $y$ & 119.0 & 1.5 \\
B & $x$ & 199.1 & 2.4 \\
& $y$ & 199.1 & 2.4 \\
C & $x$ & 597.7 & 7.3 \\
& $y$ & 597.7 & 7.3 \\
\hline
\end{tabular}

\section{Transmission Electron Microscope}

Traceability has been mainly ensured through nanodimensional standards calibrated to the international system of units (SI) traceable in industrial fields. NMIs have mainly developed AFMs equipped with laser interferometers (metrological AFMs) to calibrate nanodimensional standards. Metrological AFMs have ensured traceability to SI by calibrating the frequencies of the light sources of laser interferometers. However, the miniaturization of nanodimensional standards is progressing rapidly, and the uncertainty in the calibration of the nanodimensional standards is not sufficiently small due to the uncertainty derived from the metrological AFMs, such as the periodic error of the laser interferometer [36]. This limit has become apparent in the fact that only laser frequencies have been recommended as Mise en pratique (MeP) for the definition of the meter in the SI. The International Committee for Weights and Measures (CIPM), Consultative Committee for Length (CCL) recommended the value of Si lattice parameters as MeP for the definition of the meter in the SI in 2019 [5, 37-40]. Three guidelines for secondary realizations were also proposed in 2019 as follows.

- CCL-GD-MeP-1: Secondary realization of the SI meter using silicon lattice parameter and X-ray interferometry for nanometer- and subnanometer-scale applications in dimensional nanometrology

- CCL-GD-MeP-2: Secondary realization of SI meter using silicon lattice and transmission electron microscopy for dimensional nanometrology

- CCL-GD-MeP-3: Secondary realization of SI meter using the height of the monoatomic steps of crystalline silicon surfaces

The technical background of the CCL-GD-MeP-2 is briefly explained as follows. Techniques such as X-ray scattering can provide a link to the Si lattice spacing for a particular measurement (particularly film thickness) defined over a wide sampling area. However, various types of transmission electron microscopes appear to be the most suitable binding method for highly localized measurements of specific nanostructures. Both NIST (NMI in the US) and PTB (NMI in Germany) have made significant efforts in dimensional nanometrology that rely on this approach. The NIST Single Crystal Critical Dimension Reference Material project was a multigenerational effort to develop standards for linewidth measurements below $100 \mathrm{~nm}$ [6, 41-44]. The purpose of this project was to establish traceable linewidth measurements for specific crystalline Si nanostructures. In such measurements, the measurement target is localized by a unique mutual navigation index. Only native $\mathrm{Si}$ oxide is present on the Si structure used in such measurements. A common approach is to use a critical dimensional atomic force microscope (CD-AFM) as a comparator between those structures cross-sectioned for TEM and those that remain. The expanded uncertainty using this common approach is 
$0.6 \mathrm{~nm}(k=2)$. However, the standard samples distributed to users expanded the uncertainty $(k=2)$ between 1.5 and $2 \mathrm{~nm}$. Using TEMs to achieve this overall approach and traceability has been generally accepted in the field of dimensional nanometrology, especially in semiconductor metrology. In fact, vendors with secondary standards for the industry have introduced a similar approach to calibrate one of their products [45]. PTB has independently implemented conceptually relevant methodologies [46-48]. The PTB implementation of CD-AFM is based on a method known as Vector Approach Probing. The final uncertainties in implementing NIST and PTB were found to be similar. However, the potential for outliers suggests that the PTB method can potentially extend to even lower uncertainties. PTB, in collaboration with commercial standard sample vendors, has developed a standard sample for commercially available crystalline silicon linewidth standards.

Unfortunately, these previous studies and CD-S/TEM are not easily applicable for measuring subnanometerscale hyperfine structures. Measurement uncertainty must be reduced to at least the picometric scale to achieve such measurements. The TEM measurement target is limited to the CDs of materials containing Si single crystals because the magnification calibration is performed through direct image calibration [39]. Ordinal measurements using TEM require calibration (transfer image calibration [39]) followed by the replacement of reference material (such as a crystalline Si sample) with the desired sample. However, in TEM measurements, sample replacement (i.e., when the sample holder is removed from the TEM sample chamber and reintroduced into the TEM sample chamber and then the alignments of the electromagnetic lenses are adjusted) can cause variations with respect to magnifications calibrated based on the reference material [39]. The magnification variation of TEM images is considered to be a major source of uncertainty in TEM measurements. Therefore, suppressing this magnification fluctuation is important for realizing subnanometer measurement. Furthermore, revealing the major elements of magnification variation in the TEM images of subnanometer-scale hyperfine structures is essential.

We experimentally evaluated the measurement uncertainty due to the magnification variation of the TEM image by the instrument and the measurement error of the magnification calibration by comparing the measurements of the Si lattice spacing $d 220$ under various conditions [19]. An example TEM image of Si (220) is shown in Fig. 6. The uncertainty components evaluated were interday magnification variation, intraday repeatability, the rotation of the TEM image by $90^{\circ}$, specimen exchange, error in the incident electron beam direction, defocusing, the magnetic hysteresis of the objective lens, the magnetic hysteresis of the intermediate lenses, projection lens distortion, quantization error, and cosine error. Table 3 shows the results of the

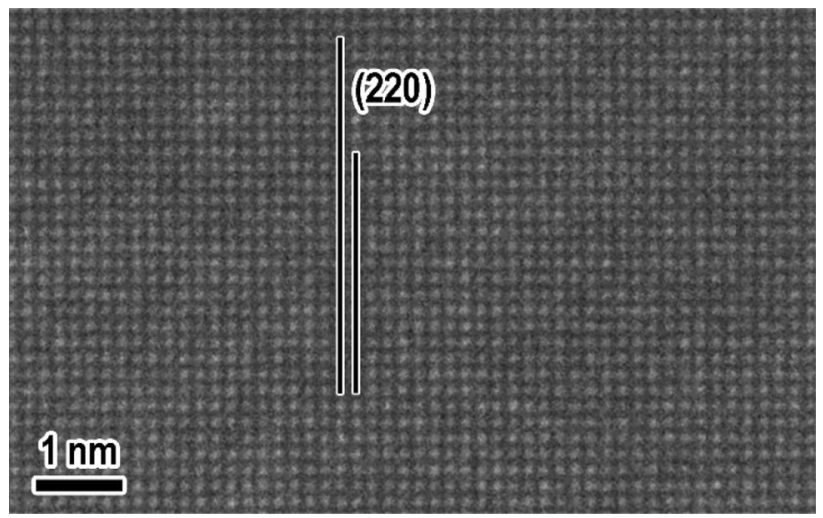

Fig. 6 TEM image of Si (220)

Table 3 Evaluation results of the uncertainty for the measured $d 220$ values of Si crystal due to the TEM instrument (JEOL, JEM-3000F) and measurement errors

\begin{tabular}{ll}
\hline Name of uncertainty component & $\begin{array}{l}\text { Uncertainty } \\
\text { component }\end{array}$ \\
\hline Interday magnification variation & 0.27 pixels \\
Intraday repeatability & 0.08 pixel \\
Rotation of TEM image by $90^{\circ}$ & 0.05 pixels \\
Specimen exchange & 0.03 pixels \\
Error in incident electron beam direction & Negligible \\
Defocusing & 0.04 pixels \\
Magnetic hysteresis of the objective lens & 0.03 pixels \\
Magnetic hysteresis of intermediate lenses & 0.12 pixels \\
Projection lens distortion & 0.08 pixels \\
Quantization error & 0.06 pixels \\
Cosine error & Negligible \\
Combined standard uncertainty & 0.33 pixels \\
Expanded uncertainty (coverage factor, $k=2)$ & 0.66 pixels \\
\hline
\end{tabular}

uncertainty evaluation. The uncertainty evaluation revealed that the change in magnification over time, magnetic history of the intermediate lens, and aberration of the projection lens are the major sources that give uncertainty to the measured value.

In the future, we will analyze changes in the lattice spacing distance caused by silicon thin film processing. Further, we will develop an absolute length measurement technology for the lattice plane spacing distance via the tilted-mAFM.

\section{Sample Preparation Technology}

The need to accurately measure the size distribution of nanoparticles is increasing to meet the new regulations that are being implemented [49]. For example, dynamic light scattering $[50,51]$ provides good ensemble data for particle size 
as long as the size distribution is narrow. For samples with a wide size distribution, although the number of observed particles is often too small to provide high-quality statistical data, microscopy (electron microscopy $[52,53]$ or atomic force microscopy [54]) is a reliable way to estimate the distribution. This chapter focuses on providing a means to produce sufficient-quality nanoparticle samples for microscopy.

When the suspension is dropped onto the substrate, nanoparticles are deposited in a ring (coffee ring effect [55]). Because the particles are stacked in a ring, identifying the isolated particles needed to obtain a reliable value for the particle size is difficult. In this case, the coffee ring effect can be avoided by vertical colloidal deposition [56]; however, horizontal particle separation cannot be achieved. Separating particles is essential for obtaining an accurate size distribution of nanoparticles using a microscope.

Nanoparticle-specific deposition methods have already been determined. For example, gold nanoparticles can be adsorbed onto a silicon substrate in a nearly isolated manner by simply immersing the substrate in a suspension [57]. However, there is often no guarantee that the original size distribution will be maintained during such a process, and we are currently looking for a common (physical rather than chemical) method that can be used for all types of nanoparticles. One of the most effective physical methods for depositing isolated nanoparticles is electrospray ionization [58]; however, it is not clear whether this process maintains the size distribution. Therefore, a more useful way to clearly maintain the size distribution of the original suspension is necessary.

Freeze-drying is the most intuitive method to obtain a sample that retains the original particle size distribution in water dispersion in all microscopic areas. However, when frozen, the nanoparticles agglomerate. In the pharmaceutical industry, chemicals are often added to suspensions to raise the glass transition temperature of the solvent [59] and immobilize nanoparticles at high temperatures. Although this technique works well, the nanoparticles are embedded under the dried chemicals during drying. Therefore, such samples are unsuitable for microscopic observation. For atomic force microscopy, the situation is exacerbated because the dry chemicals on the substrate make it almost impossible to determine the height of the substrate. Without adding chemicals, the low thermal conductivity and high heat of solidification of water give the nanoparticles in the suspension sufficient time to agglomerate. The freezing process by preparing a thin film of suspension was selected to increase the speed. Sample pretreatment with thin films also has the advantage of being able to create the two-dimensional samples needed for microscopy from the beginning.

In contrast to the biological case [60], water does not need to be in a glassy state during freezing. Liquid nitrogen temperature is not required, but such low temperatures are useful
(1)

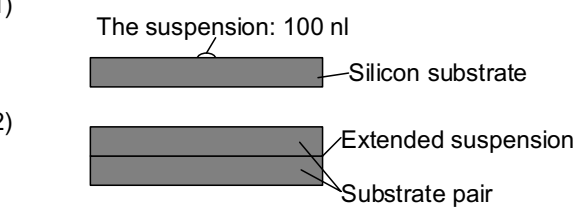

(3)

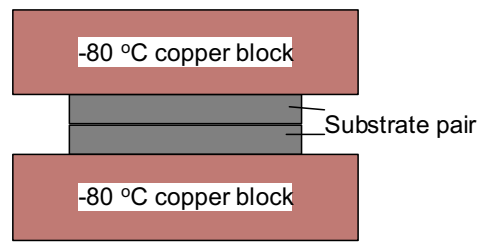

(4)

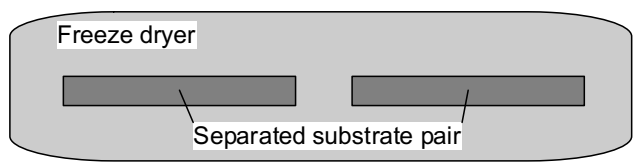

Fig. 7 Procedure for the sandwich freeze-drying method

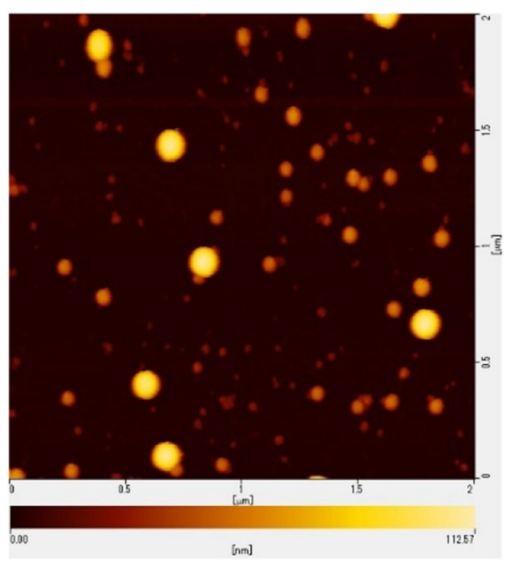

Fig. 8 AFM image of a sample prepared through the sandwich freeze-drying method. (Polystyrene latex nanoparticles with diameters of 100,50 , and $25 \mathrm{~nm}$ )

for obtaining sufficient freezing rates. The freezing temperature of $-80{ }^{\circ} \mathrm{C}$ was selected because it is easily available in conventional freezers. Because silicon wafers are widely used for microscopic measurements, despite their low thermal conductivity, they were used as substrates. Thus, a method of rapidly freezing the dispersion under these conditions is necessary.

Figure 7 shows the procedure of the sandwich freezedrying method [26, 51]. (1) A 100-nl suspension is placed on a 1-cm-square silicon substrate. (2) The suspension is extended between the silicon substrate pair. (3) The sandwich substrate is packed between the copper blocks at $-80{ }^{\circ} \mathrm{C}$. (4) The substrate pair is separated with tweezers. After performing these steps, the substrates are placed in a container and connected to a lyophilizer to dry. Figure 8 shows an example of an AFM image of a sample 
(polystyrene latex nanoparticles with diameters of 100, 50, and $25 \mathrm{~nm}$ ) prepared through the sandwich freeze-drying method. It can be observed in the figure that the particles are individually fixed without agglomeration.

\section{Summary}

We described the current status of the research and development of nanodimensional standards technology at the NMIJ in AIST. In the future, we will expand the calibration service range of the step height measurement using metrological AFM and start a calibration service of TEM magnification calibration samples. We also plan to distribute the next CRM for SEM image sharpness evaluation. We will continue to develop increasingly accurate nanodimensional standards.

Acknowledgements This work was partially supported by the New Energy and Industrial Technology Development Organization (NEDO) (Project code P09002), JSPS KAKENHI Grant Number JP16K18119, and the Japan Science and Technology Agency, JSPS KAKENHI Grant Number 20K04511. This study was partly related to the studies conducted by the Consortium for Measurement Solutions for Industrial Use of Nanomaterials (COMS-NANO) in Japan.

Conflict of interest The authors declare no conficts of interest.

Open Access This article is licensed under a Creative Commons Attribution 4.0 International License, which permits use, sharing, adaptation, distribution and reproduction in any medium or format, as long as you give appropriate credit to the original author(s) and the source, provide a link to the Creative Commons licence, and indicate if changes were made. The images or other third party material in this article are included in the article's Creative Commons licence, unless indicated otherwise in a credit line to the material. If material is not included in the article's Creative Commons licence and your intended use is not permitted by statutory regulation or exceeds the permitted use, you will need to obtain permission directly from the copyright holder. To view a copy of this licence, visit http://creativecommons.org/licenses/by/4.0/.

\section{References}

1. Meli F, Thalmann R (1998) Long-range AFM profiler used for accurate pitch measurements. Meas Sci Technol 9:1087

2. Buhr E, Michaelis W, Diener A, Mirandé W (2007) Multi-wavelength VIS/UV optical diffractometer for high-accuracy calibration of nano-scale pitch standards. Meas Sci Technol 18:667

3. Dai G, Pohlenz F, Dziomba T, Xu M, Diener A, Koenders L, Danzebrink H-U (2007) Accurate and traceable calibration of two-dimensional gratings. Meas Sci Technol 18:415

4. Bienias M, Gao S, Hasche K, Seemann R, Thiele K (1998) A metrological scanning force microscope used for coating thickness and other topographical measurements. Appl Phys A 66:S837

5. Garnaes J, Necas D, Nielsen L, Madsen MH, Torras-Rosell A, Zeng G, Klapetek P, Yacoot A (2020) Algorithms for using silicon steps for scanning probe microscope evaluation. Metrologia 57:064002

6. Dixson RG, Allen RA, Guthrie WF, Cresswell MW (2005) Traceable calibration of critical-dimension atomic force microscope linewidth measurements with nanometer uncertainty. J Vac Sci Technol B 23:3028

7. Orji NG, Dixson RG (2007) Higher-order tip effects in traceable CD-AFM-based linewidth measurements. Meas Sci Technol $18: 448$

8. Garnaes J, Kofod N, Kuehle A, Nielsen C, Dirscherl K, Blunt L (2003) Calibration of step heights and roughness measurements with atomic force microscopes. Precis Eng 27:91

9. Meli F, Klein T, Buhr E, Frase CG, Gleber G, Krumrey M, Duta A, Duta S, Korpelainen V, Bellotti R, Picotto GB, Boyd RD, Cuenat A (2012) Traceable size determination of nanoparticles, a comparison among European metrology institutes. Meas Sci Technol 23:125005

10. Lin H-L, Fu W-E, Weng H-F, Misumi I, Sugawara K, Gonda S, Takahashi K, Takahata K, Ehara K, Takatsuji T, Fujimoto T, Salas J, Dirscherl K, Garnas J, Damasceno J, Oliveira JCV, Emanuele E, Picotto GB, Kim CS, Cho SJ, Motzkus C, Meli F, Gao S, Shi Y, Liu J, Jämting K, Catchpoole HJ, Lawn MA, Herrmann J, Coleman VA, Adlem L, Kruger OA, Buajarern J, Buhr E, Danzebrink H-U, Krumrey M, Bosse H (2019) Nanoparticle characterization - supplementary comparison on nanoparticle size. Metrologia 56(1):04004

11. Misumi I (2021) Nanoscale standards by metrological AFM and other instruments. IOP Books, Online ISBN: 978-0-7503-31913, Print ISBN: 978-0-7503-3189-0

12. Gonda S, Doi T, Kurosawa T, Tanimura Y, Hisata N, Yamagishi T, Fujimoto H, Yukawa H (1999) Real-time, interferometrically measuring atomic force microscope for direct calibration of standards. Rev Sci Instrum 70:3362

13. Misumi I, Gonda S, Huang Q, Keem T, Kurosawa T, Fujii A, Hisata N, Yamagishi T, Fujimoto H, Enjoji K, Aya S, Sumitani H (2005) Sub-hundred nanometre pitch measurements using an AFM with differential laser interferometers for designing usable lateral scales. Meas Sci Technol 16:2080

14. Kizu R, Misumi I, Hirai A, Kinoshita K, Gonda S (2018) Development of a metrological atomic force microscope with a tiptilting mechanism for 3D nanometrology. Meas Sci Technol 29:075005

15. Itoh H, Fujimoto T, Ichimura S (2006) Tip characterizer for atomic force microscopy. Rev Sci Instrum 77:103704

16. Sigehuzi T (2017) Depositing nanoparticles on a silicon substrate using a freeze drying technique. J Chem Phys 147:084201

17. Kumagai K, Kurokawa K (2020) Development of NMIJ CRM 5207-a tungsten dot-array for the image sharpness evaluation in scanning electron microscopy - structure evaluation and determination of dot-pitch. Microscopy 69:360

18. Kumagai K, Kurokawa K (2021) Evaluation of image distortion in SEM by using a dot-array-based certified reference material. Microscopy 70:353

19. Kobayashi K, Misumi I, Yamamoto K (2021) Experimental evaluation of uncertainty in sub-nanometer metrology using transmission electron microscopy due to magnification variation. Meas Sci Technol 32:095011

20. National Metrology Institute of Japan (2018) NMIJ CRM 5207-a Tungsten Dot-array (National Metrology Institute of Japan, Japan). 25 March 2020, https://unit.aist.go.jp/nmij/english/refma te/crm/cert/5207a_en.pdf

21. ISO 13095 (2014) Surface chemical analysis - atomic force microscopy - procedure for in situ characterization of AFM probe shank profile used for nanostructure measurement

22. ISO 16700 (2016) Microbeam analysis - scanning electron microscopy - guidelines for calibrating image magnification

23. Sugawara K, Sato O, Yoshizaki K, Misumi I, Gonda S, Takatsuji T, Kitta J (2006) Effects of angle misalignments on pitch measurements of two-dimensional gratings using an AFM. J Chin Soc Mech Eng 27:531 
24. Misumi I, Gonda S, Kurosawa T, Azuma Y, Fujimoto T, Kojima I, Sakurai T, Ohmi T, Takamasu K (2006) Reliability of parameters of associated base straight line in step height samples: uncertainty evaluation in step height measurements using nanometrological AFM. Precis Eng 30:13

25. Misumi I, Naoi K, Sugawara K, Gonda S (2015) Profile surface roughness measurement using metrological atomic force microscope and uncertainty evaluation. Measurement 73:295

26. Kizu R, Misumi I, Hirai A, Kinoshita K, Gonda S (2019) Linewidth calibration using a metrological atomic force microscope with a tip-tilting mechanism. Meas Sci Technol 30:015004

27. NanoCD Standards (NCD), VLSI Standards Inc. https://www.vlsis tandards.com/products/dimensional/nanocd.asp?sid $=83$

28. Sato M, Orloff J (1991) A method for calculating the current density of charged particle beams and the effect of finite source size and spherical and chromatic aberrations on the focusing characteristics. J Vac Sci Technol B 9:2602-2608

29. Kratschmer E, Rishton SA, Kern DP, Chang THP (1988) Quantitative analysis of resolution and stability in nanometer electron beam lithography. J Vac Sci Technol B 6:2074-2079

30. Joy DC (2002) SMART - a program to measure SEM resolution and imaging performance. J Microsc 208:24-34

31. Lorusso GF, Joy DC (2003) Experimental resolution measurement in critical dimension scanning electron microscope metrology. Scanning 25:175-180

32. Ishitani T, Sato M (2007) Evaluation of both image resolution and contrast-to-noise ratio in scanning electron microscopy. J Electron Microsc 56:145-151

33. Joy DC, Ko YU, Hwu JJ (2000) Metrics of resolution and performance for CD-SEMs. In: Sullivan NT (ed) Metrology, inspection, and process control for microlithography. SPIE-Int Soc Optical Engineering, Bellingham, pp 108-114

34. ISO/TS 24597:2011 Microbeam analysis — scanning electron microscopy - methods of evaluating image sharpness

35. Matthews M, Shah J (2015) Testing of the DR method for image sharpness determination. Microsc Microanal 21(S3):2239-2240

36. Misumi I, Sugawara K, Kizu R, Hirai A, Gonda S (2019) Extension of the range of profile surface roughness measurements using metrological atomic force microscope. Prec Eng 56:321

37. Yacoot A, Bosse H, Dixson R (2020) The lattice parameter of silicon: a secondary realisation of the metre. Meas Sci Technol 31:121001

38. Yacoot A, Kuetgens U, Massa E, Dixson R and Bosse H (2019) Recommendations of CCL/WG-N on: realization of the SI meter using silicon lattice parameter and x-ray interferometry for nanometer and sub-nanometer scale applications in dimensional nanometrology, CCL-GD-MeP-1

39. Dixson R, Bosse H, Yacoot A (2019) Recommendations of CCL/ WG-N on: Realization of SI metre using silicon lattice and Transmission Electron Microscopy for dimensional nanometrology, CCL-GD-MeP-2

40. Koenders A L, Busch I, Garnaes J, Yacoot A, Dixson R and Bosse $\mathrm{H}$ (2019) Recommendations of CCL/WG-N on: Realization of SI metre using height of monoatomic steps of crystalline silicon surfaces, CCL-GD-MeP-3

41. Cresswell MW, Bogardus EH, de Pinillos JVM, Bennett MH, Allen RA, Guthrie WF, Murabito CE, Am Ende BA, Linholm LW (2002) CD Reference materials for sub-tenth micrometer applications. Proc SPIE 4689:116

42. Cresswell M, Guthrie W, Dixson R, Allen RA, Murabito CE, Martinez de Pinillos JV (2006) RM8111: development of a prototype linewidth standard. J Res Natl Inst Stand Technol 111:187
43. Orji NG, Badaroglu M, Barnes BM, Beitia C, Bunday BD, Celano U, Kline RJ, Neisser M, Obeng Y, Vladar AE (2018) Metrology for the next generation of semiconductor devices. Nature Electron $1: 532$

44. Orji NG, Dixson RG, Garcia-Gutierrez DI, Bunday BD, Bishop M, Cresswell MW, Allen RA, Allgair JA (2016) TEM calibration methods for critical dimension standards. J Micro/Nanolith MEMS MOEMS 15:044002

45. Tortonese M, Lorusso G, Blanquies R, Prochazka J, Grella L (2004) Sub-50-nm isolated line and trench width artifacts for CD metrology. Proc SPIE 5375:647

46. Dai G, Heidelmann M, Kübel C, Prang R, Fluegge J, Bosse H (2013) Reference nano-dimensional metrology by scanning transmission electron microscopy. Meas Sci Technol 24:085001

47. Dai G, Zhu F, Heidelmann M, Fritz G, Bayer T, Kalt S, Fluegge J (2015) Development and characterisation of a new linewidth reference material. Meas Sci Technol 26:115006

48. Dai G, Koenders L, Fluegge J, Bosse H (2016) Two approaches for realizing traceability in nanoscale dimensional metrology. Opt Eng 55:091407

49. EU Commission Recommendation of 18 October 2011 on the definition of nanomaterial

50. Wyatt P (1998) Submicrometer particle sizing by multiangle light scattering following fractionation. J Colloid Interface Sci 197:9

51. Takahashi K, Kramar JA, Farkas N, Takahata K, Misumi I, Sugawara K, Gonda S, Ehara K (2019) Interlaboratory comparison of nanoparticle size measurements between NMIJ and NIST using two different types of dynamic light scattering instruments. Metrologia 56:055002

52. Akita T, Kohyama M, Haruta M (2012) Electron microscopy study of gold nanoparticles deposited on transition metal oxides. Acc Chem Res 46:1773

53. Kumagai K, Kurokawa A (2019) Measurement of the number concentration of gold nanoparticle suspension by scanning electron microscopy. Metrologia 56:044001

54. Misumi I, Sugawara K, Takahata K, Takahashi K, Ehara K (2018) Size measurements of standard nanoparticles using metrological atomic force microscope and evaluation of their uncertainties. Precis Eng 51:691-701

55. Deegan RD, Bakajin O, Dupont TF, Huber G, Nagel SR, Witten TA (1997) Capillary flow as the cause of ring stains from dried liquid drops. Nature 389:827

56. Diao JJ, Sun J, Hutchison JB, Reeves ME (2005) Self-assembled nanoparticle wires by discontinuous vertical colloidal deposition. Appl Phys Lett 87:103113

57. Bossard-Giannesini L, Cruguel H, Lacaze E, Pluchery O (2016) Plasmonic properties of gold nanoparticles on silicon substrates: understanding Fanolike spectra observed in reflection. Appl Phys Lett 109:111901

58. Therrien J, Dindar A, Smith D (2007) AFM studies of nanoparticle deposition via electrospray ionization. Microsc Res Tech 70:530

59. Abdelwahed W, Degobert G, Stainmesse S, Fessi H (2006) Freeze-drying of nanoparticles: formulation, process and storage considerations. Adv Drug Delivery Rev 58:1688

60. Fujimoto T, Fujimoto K (1997) Metal sandwich method to quickfreeze monolayer cultured cells for freeze-fracture. J Histochem Cytochem 45:595 